\title{
Is Surgical Dermatology a fiction due to the existence of Plastic and Aesthetic Surgery and Cosmetology, and who is to perform these operations?
}

\author{
Radmilo Roncevic \\ Clinic for Plastic and Reconstructive Surgery, Clinical Center of Serbia, Belgrade, Serbia
}

$\mathrm{T}$

This question is not uncommonly asked, especially by surgeons who deal with less complex procedures in the field of plastic and reconstructive surgery. The reason for these questions, among others, is probably due to direct competition.

As a surgeon who performs extensive plastic and craniofacial surgery and surgery of the orbit, and who is not in competition with doctors dealing with surgical dermatology but knows the procedures in the field of surgical dermatology, I could, perhaps impartially, try to answer these questions.

Surgical dermatology is not a fiction but a real need which has improved some areas in which plastic surgery, or "big surgery", has not shown more interest, often leaving some things from the domain of "small surgery" neglected and inadequately treated.

Surgical dermatology is often the domain of physicians and dermatologists who specialize in skin surgery, as well as specialists in plastic and aesthetic surgery. Which of the mentioned specialties is closest to this surgical discipline, in whose hands is the future of this surgery, and who is the real "owner" of this surgery? I would say: dermatologists who specialize in skin surgery. Why? Dermatologists, compared to the other mentioned specialists, are more familiar with the anatomy, physiology and pathology of the skin as well as the diagnostics, especially the differential diagnosis of numerous pathological conditions of the skin. These are the basics without which it is impossible to start any treatment or any therapeutic procedure. Specialists in plastic and cosmetic surgery dealing with this surgery in their core specialty most often deal with less complex and less extensive procedures. On the other side, usually ambitious and creative dermatologists perform surgical dermatology, too. Ambitious and creative personalities in every profession, with their daily work and scientific achievements, contribute to the advancement of their profession. Dermatologic surgeons are pioneers in the research and development of many of today's most effective therapeutic and cosmetic skin care solutions.

Surgical dermatology applies surgical/bloody and many non-surgical/bloodless procedures. It is often possible to treat a pathological condition surgically or by a non-surgical procedure. Both methods have their advantages and disadvantages. In any particular case, the procedure should be decided based on which will give the best result. Unfortunately some doctors, who are not sufficiently trained for a certain procedure that would give a better result, sometimes apply less efficient procedures.

The most prevalent view today is that in cases of basal cell carcinoma, which usually appears on the face, surgical excision therapy is significantly more efficient than radiation therapy or other nonsurgical procedures, and that recurrences after radiation therapy are significantly more malignant than recurrences occurring after excision. Postradiation recurrences of basal cell carcinoma are often very aggressive, invasive (carcinoma basocellulare terebrans/ulcus terebrans) practically incurable. Nonetheless, doctors who are not trained for radical excision of the tumor or in successful reconstruction of the post-excision defect often refer such patients to radiation therapy. In cases of malignant melanoma, due to the doctors' insufficient surgical training, it is a common mistake to not perform radical excision or to apply an inadequate therapeutic procedure. Often the mini facelift is applied inappropriately; mini lifting of the face should not be performed in cases where only extensive superficial muscular aponeurotic system (SMAS) lifting can give good results.

Errors are often committed in the implementation of dermabrasion. Of course, microdermabrasion and super-

Copyright ( 2017 Roncevic R. This is an Open Access article distributed under the terms of the Creative Commons Attribution-NonCommercial 4.0 International License (http://creativecommons.org/licenses/by-nc/4.0/), permitting all non-commercial use, distribution, and reproduction in any medium, provided the original work is properly cited. 
ficial dermabrasion give good results in certain cases. But in some cases, such as deep scars after acne and deep tattoos, these procedures cannot produce an evident result. In cases of deep scars after acne or other deeper changes to the skin, in order to obtain a good and evident result, it is necessary to do deep dermabrasion to remove the epidermis and dermis to the level of skin ablation for deep intermediate skin graft (intermedial Blair II free skin transplant in the reticular layer of the dermis). From the rest of the reticular layer of the dermis and the rest of the epithelial cells of sebaceous glands, sweat glands and hair roots, skin regeneration will follow and scars will be much shallower. If needed, after a while, the abrasion can be repeated. Inexperienced surgeons avoid this procedure and thus do not achieve good results. The situation is similar in the event of laser treatment.

So, one of the possible significant problems and shortcomings in the practical application of surgical dermatology, surgical and nonsurgical procedures is the lack of radicalism in the implementation of certain procedures. This particularly applies to the use of surgical procedures. The radicalism is often avoided because of compromises with aesthetics. Unfortunately, these compromises are often later the cause of major aesthetic problems. In order for doctors who deal with surgical dermatology to accept radical procedures as a necessary part of the profession they are involved in, they should occasionally stay in the surgery departments where they can perform large, radical surgical procedures.

Regardless that surgical dermatology represents "small surgery", there are numerous possible errors with large, even dangerous, consequences. Therefore, continuing medical education is the "conditio sine qua non" for this young, new surgical discipline.

Like the entire medicine, surgical dermatology is under constant pressure from aggressive pharmaceutical and cosmetic industries that are trying to realize their profits through illegal and unethical practices, even with the aid from some doctors. In recent years, surgical dermatology is one of the medical disciplines that is most exposed to this pressure. Will we resist the pressure and preserve the reputation and ethics of the profession?

Keywords: Surgical dermatology; surgical procedures; non-surgical procedures

Citation: Roncevic R, Is Surgical Dermatology a fiction due to the existence of Plastic and Aesthetic Surgery and Cosmetology, and who is to perform these operations? J Surg Dermatol 2017; 2(1): 5-6; http://dx.doi.org/10.18282/ jsd.v2.i1.117.

Received: $5^{\text {th }}$ December 2016; Published Online: $25^{\text {th }}$ January 2017

Correspondence to: Radmilo Roncevic, Clinic for Plastic and Reconstructive Surgery, Clinical Center of Serbia, Belgrade, Serbia, ronac@eunet.rs. 